\title{
Pregabalin als seltene Ursache einer Hepatopathie
}

Michael Kowar, Claudia Friedrich, Andreas H. Jacobs

Das GABA-Analogon Pregabalin wird bei Epilepsien, neuropathischen Schmerzen und generalisierten Angststörungen verordnet [1]. In den letzten Jahren hat die Zahl der PregabalinVerordnungen zugenommen, mit einem weiteren Anstieg ist zu rechnen [2, 3]. Damit dürften auch ungewöhnliche Nebenwirkungen zunehmen - ein Beispiel zeigt der geschilderte Fall.

Anamnese I Eine 76-jährige Patientin wird aufgrund zunehmender, immobilisierender Schmerzen in der unfallchirurgischen Abteilung aufgenommen. Eine MRT-Untersuchung zeigt eine Fraktur des Os sacrum, die auf vorherige Stürze zurückging. Nach konservativer Therapie wird die Patientin zur weiteren Mobilisation im Rahmen einer frührehabilitativen Therapie in unsere geriatrische Abteilung verlegt. Sie hat folgende Vorerkrankungen:

- Hypothyreose nach subtotaler Strumektomie,

- arterieller Hypertonus und

- Cox-Arthrose.

Körperlicher Untersuchungsbefund | Die Patientin ist in reduziertem Allgemein-, aber normalem Ernährungszustand ( $56 \mathrm{~kg}$ ). Die internistische Untersuchung bringt folgende Befunde:

- Eupnoe,

- vesikuläres Atemgeräusch,

- Herztöne rein und rhythmisch,

- keine Ödeme,

- kein Sklerenikterus,

- Abdomen weich mit lebhaften Darmgeräuschen und ohne Abwehrspannung.

Bei der neurologischen Untersuchung zeigt sich ein unsicheres Gangbild mit ungerichteter Fallneigung. Hinweise auf Paresen oder sensible Ataxie fehlen, Orientierung, Mnestik und Stimmung sind altersgerecht.
Labordiagnostik | Die Infektparameter sind leicht erhöht:

- C-reaktives Protein 0,67 mg/dl

- Lactatdehydrogenase 265U/1

- alkalische Phosphatase $191 \mathrm{U} / 1$

Da die Werte aber im Vergleich zum Aufenthalt in der unfallchirurgischen Abteilung bereits gefallen sind, führen wir sie auf die Fraktur zurück. Die weiteren Laborwerte liegen im Referenzbereich.

Medikation I Die Gangunsicherheit führen wir auf die Schmerzsymptomatik zurück. Bereits in der unfallchirurgischen Abteilung erfolgte eine Analgesie nach WHO-Stufenschema mit Ibuprofen, Metamizol und Tilidin/Naloxon, das die Patientin prästationär gut vertragen hat. Aufgrund des ungünstigen Nebenwirkungsprofils im Alter setzten wir das Ibuprofen am Verlegungstag ab. Die Thromboseprophylaxe wird mit Enoxaparin fortgeführt. Zudem nimmt die Patientin seit dem operativen Eingriff Kalzium-BrauseTabletten ein.

Radikuläre Schmerzen | Unter weiterführender Krankengymnastik persistieren die Schmerzen und die Gangunsicherheit. Da die Beschwerden in das rechte Bein ausstrahlen (Ausbreitungsgebiet L5/S1), erhält die Patientin Pregabalin:

- zunächst $25 \mathrm{mg}$ am Abend an Tag 0

- schrittweise erhöht auf $75 \mathrm{mg}$ an Tag 8 .
Abb. 1 Verlauf der Leberwerte. Links: Bilirubin; rechts: GOT, GPT, GGT, Ammoniak, AP und INR

a) Absetzen von Pregabalin

b) Verlegung Rehabilitationsklinik

c) hausärztliche Kontrolle.
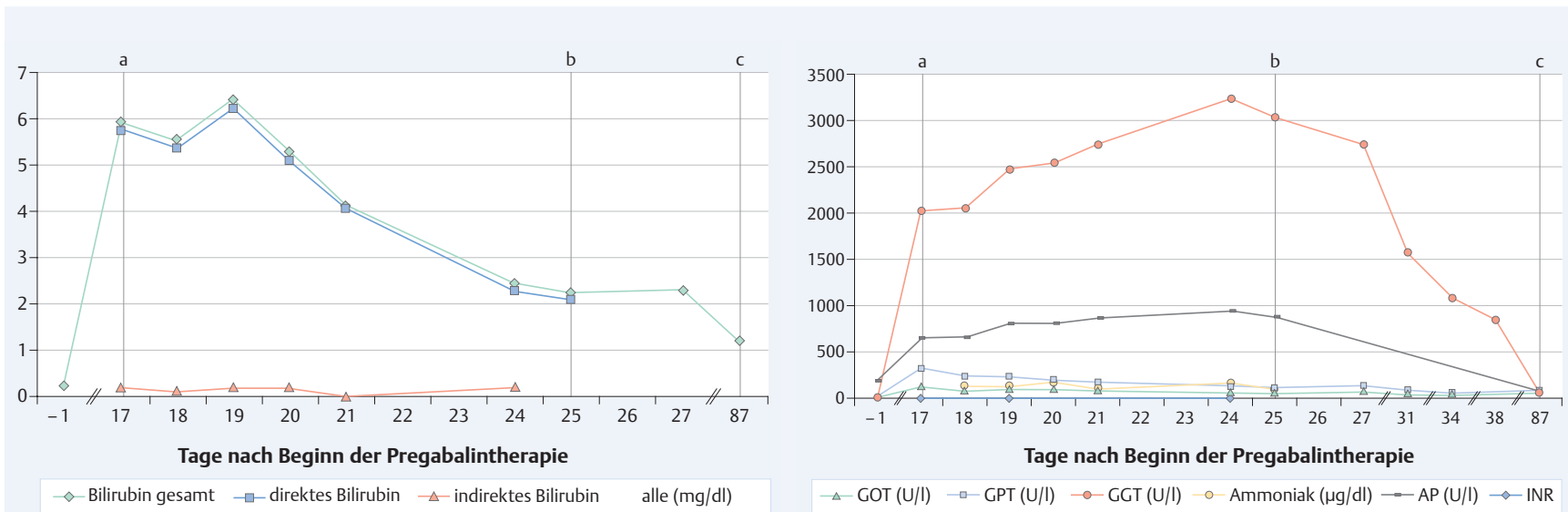


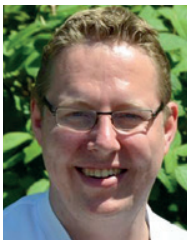

Dr. med. Michael Kowar ist Oberarzt an der Klinik für Geriatrie mit Neurologie und Tagesklinik, Johanniter Krankenhaus, Bonn. michael.kowar@johanniterkliniken.de

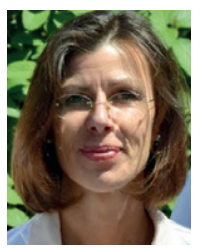

Claudia Friedrich ist Oberärztin an der Klinik für Geriatrie mit Neurologie und Tagesklinik, Johanniter Krankenhaus, Bonn. claudia.friedrich@johanniterkliniken.de

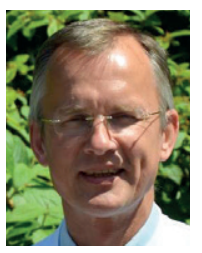

Prof. Dr. med.

Andreas $\mathrm{H}$. Jacobs

ist Chefarzt der Klinik für Geriatrie mit Neurologie und Tagesklinik, Johanniter Krankenhaus, Bonn. andreas.jacobs@johanniterkliniken.de
Gastrointestinale Beschwerden I An Tag 10 beginnt die Patientin über Übelkeit zu klagen. Klinisch ist das Abdomen weich mit regelrechten Darmgeräuschen, ohne Resistenzen oder Druckschmerz. Zum Erbrechen kommt es nicht. Die Therapie mit Kalzium-Brause wird auf Vitamin- $D_{3}$-Kapseln umgestellt. Da die Patientin zu diesem Zeitpunkt konstipiert ist, verabreichen wir Abführmittel wie Lexicarbon Suppositorium, Lactulose und Glycerin Suppositorium. Hierunter normalisiert sich der Stuhlgang. Trotzdem persistiert die Übelkeit.

Schlechte Leberwerte I Am Tag 15 kommt es zu einem Sklerenikterus, so dass eine laborchemische Kontrolle erfolgt ( Abb. 1). Deutlich erhöht sind:

- Bilirubin $6 \mathrm{mg} / \mathrm{dl}$

- Glutamat-Oxalazetat-Transaminase $131 \mathrm{U} / \mathrm{I}$

- Glutamat-Pyruvat-Transaminase 2030U/I

- alkalische Phosphatase 650U/I

- Ammoniak $128 \mu \mathrm{g} / \mathrm{dl}$

- C-reaktives Protein $4 \mathrm{mg} / \mathrm{dl}$

Die Abdomensonografie gibt keinen Hinweis auf eine Cholestase.

Weitere Therapie I Die Therapie mit Pregabalin, Enoxaparin und Tilidin/Naloxon wird beendet. Die Patientin erhält:

- Aminosteril Hepa,

- Ceftriaxon (V.a. unklares Infektgeschehen) und

- Prednisolon (V.a. eine autoimmune Genese).

Die Hepatitis-Serologie bleibt negativ. Laborchemisch gibt es keinen Hinweis auf eine immunogene Genese - antimitochondriale Antikörper sind unauffällig. Daher stellen wir die Kortison-Therapie nach 5 Tagen ein. In der MR-Cholangiopankreatikografie zeigt sich ebenfalls kein pathologischer Befund, insbesondere gibt es keinen Anhalt für Cholezystitis, Choledocholithiasis, Raumforderung oder Leberzirrhose.

Besserung I Nach Medikamentenumstellung bessern sich die Leberwerte langsam, sodass von einer medikamentös induzierten Hepatopathie auszugehen ist. Die Patientin wird in eine geriatrische Rehabilitationsklinik verlegt. Hier normalisieren sich die Leberwerte im Verlauf.

\section{Diskussion}

http://dx.doi.

org/10.1055/s-0041-105987

Interessenkonflikt

Die Autoren geben an, dass kein Interessenkonflikt besteht.

DOI 10.1055/s-0041-105987

Dtsch Med Wochenschr 2015; 140: 1759-1760

(c) Georg Thieme Verlag KG . Stuttgart · New York.

ISSN 0012-0472

Nebenwirkungen von Pregabalin I Häufige Nebenwirkungen von Pregabalin sind Müdigkeit und Benommenheit. Sie waren in den Zulassungsstudien die häufigste Ursache für einen Abbruch der Therapie [4]. Daher sollte Pregabalin einschleichend und vorwiegend abends eingenommen werden. Weitere häufige Nebenwirkungen sind Nasopharyngitis, gesteigerter Appetit, Verwirrtheit, Übelkeit, periphere Ödeme, erektile Dysfunktion und Gangstörungen [4, 5].
Pregabalin-induzierten Hepatopathie I Interessanterweise wird Pregabalin nicht über die Leber abgebaut [1]. Eine Hepatopathie im Rahmen einer Therapie unter Pregabalin wird daher in der Fachinformation nicht angegeben. Auch in der Literatur (PubMed, medpilot) fanden wir nur drei Fallberichte einer Pregabalin-induzierten Hepatopathie $[6,7,8]$. In dem Fallbericht von Sendra et al. [6] zeigten sich wie bei uns 14 Tage nach Beginn einer Pregabalintherapie erhöhte Leber-Werte. Diese waren nicht so stark erhöht wie im vorliegenden Fall, allerdings hatte der Patient mit einer Hämosiderose eine hepatische Vorerkrankung.

Erhöhte INR-Werte | In einem Fall [8] bestand neben erhöhten Leberwerten auch eine eingeschränkte Lebersynthese (INR 4,2). Diese könnte ein gesteigertes Mortalitätsrisiko darstellen [9]. Bei unserer Patientin war die INR durchgehend im Normbereich.

Enoxaparin und Tilidin / Naloxon als Auslöser? | Unsere Patientin klagte über Übelkeit, die wir zunächst auf die Kalzium-Brause-Tabletten zurückführten. Trotz Therapieumstellung verschlechterte sich ihr Zustand: Es kam zu einem Ikterus. Daraufhin beendeten wir die Therapie mit den potenziell lebertoxischen Medikamenten Enoxaparin, Tilidin/Naloxon und Pregabalin. Dass Enoxaparin oder Tilidin/Naloxon die Hepatopathie ausgelöst haben, ist unwahrscheinlich: Die Patientin hatte diese bereits in der unfallchirurgischen Abteilung eingenommen, die Leberwerte bei Aufnahme in unserer Abteilung waren aber unauffällig.

Differenzialdiagnosen | Bei unserer Patientin war das direkte Bilirubin stark erhöht (5,41 mg/ dl).Wir vermuteten eine intrahepatische Cholestase und führten daher sowohl eine Sonografie als auch eine MR-Cholangiopankreatikografie durch - beide blieben ohne wegweisende Befunde. Für eine virale Hepatitis ergab sich laborchemisch kein Anhalt. Es kam auch eine autoimmune Hepatitis in Frage, weswegen wir eine Kortison-Therapie einleiteten. Da dieser Verdacht sich durch Laborwerte nicht bestätigen ließ, wurde die Therapie eingestellt. Die Hepatopathie stand in zeitlichem Zusammenhang mit Pregabalin; nach Absetzen des Präparates besserten sich die Leberwerte sukzessive.

Vollständige Ausheilung I In allen bisherigen Beobachtungen und so auch bei uns zeigte sich eine Restitutio ad integrum innerhalb von einigen Wochen nach Absetzen von Pregabalin. Fallberichte über Todesfälle sind uns nicht bekannt.
Konsequenzen für Klinik und Praxis

- Bei erhöhten Leberwerten und beginnendem Leberversagen unklarer Genese kommt eine Pregabalin-induzierte Hepatopathie in Frage, auch wenn dies in der Literatur bisher kaum beschrieben ist. 


\section{Literatur}

1 Ben-Menachem E. Pregabalin pharmacology and its relevance to clinical practice. Epilesia 2004; 45 (Suppl. 6): 13-18

2 Schwabe U, Paffrath D Hrsg. ArzneiverordnungsReport 2010. Heidelberg: Springer Medizin; 2010

3 Kassenärztliche Vereinigung Nordrhein. Pregabalin-Generika in allen Anwendungsgebieten verordnungsfähig

http://www.kvno.de/60neues/2014/pregabalin/ index.html

Letzter Zugriff am 26. 08. 2015

4 Pfizer Pharma GmbH. Fachinformation „Lyrica ${ }^{\circledR}$ Hartkapseln". Stand: Juli 2014

5 Zaccara G, Gangemi P, Perucca P, Specchio L. The adverse event profile of pregabalin: a systematic review and meta-analysis of randomized controlled trials. Epilepsia 2011; 52: 826-836

6 Sendra JM, Junyent TT, Pellicer MJ. Pregabalininduced hepatotoxicity. Ann Pharmacother 2011; 45: e32

7 Kaufmann MK, Choy M. Pregabalin and Simvastatin First Report of a Case of Rhabdomyolysis. P\&T 2012; 37: 579-581

8 Einarsdottir S, Björnsson E. Pregabalin as a probable cause of acute liver injury. Eur J Gastroenterol Hepatol 2008; 10: 1049

9 Björnsson E, Ollson R. Outcome and prognostic markers in severe drug-induced liver diseases. Hepatology 2005; 42: 481-489 